\title{
Computed-tomography volumetric study of the ethmoid labyrinth
}

\author{
Ioan Alexandru Bulescu', Octavian Munteanu', Razvan Stanciulescu', Cosmin Pantu', \\ Mihai Enyedi', Florin Filipoiu', Vlad Andrei Budu² \\ ${ }^{1}$ Department of Morphological Sciences, Discipline of Anatomy, "Carol Davila” University of Medicine \\ and Pharmacy, Bucharest, Romania \\ 2"Prof. Dr. D. Hociota” Institute for Phono-Audiology and Functional ENT Surgery, Bucharest, Romania
}

\begin{abstract}
Morphological variability of paranasal sinuses is well known for endoscopic surgeons and anatomists alike. The ethmoid sinus is the most complex and variable of all paranasal sinuses, due to the fact that its development is not yet well known and is influenced by many factors. Volumetric studies of the sinuses have been made using dried skulls, cadaver heads and imaging studies, but there are still not sufficient data in order to name a standard value for each sinus. Few data can be found especially regarding the ethmoid sinus.

In this paper, we measured the volumes of ethmoid lateral masses, and for anterior and posterior groups of cells, using imaging studies and a volumetric feature of our imaging studies.

Results showed an average volume between $7.34 \mathrm{~cm}^{3}$ and $8.39 \mathrm{~cm}^{3}$ for the ethmoid lateral mass, between $4.33 \mathrm{~cm}^{3}$ and $4.92 \mathrm{~cm}^{3}$ for the anterior ethmoid and between $3.01 \mathrm{~cm}^{3}$ and $3.47 \mathrm{~cm}^{3}$ for the posterior ethmoid groups. We also found that the average volume of the anterior ethmoid occupies between $58-59 \%$ of the whole volume, while the posterior ethmoid occupies only $41-42 \%$ of this volume.
\end{abstract}

KEYWORDS: ethmoid sinus, volume, computed-tomography

\section{INTRODUCTION}

Variability regarding the size and shape of paranasal sinuses is a well-known aspect in the field of sinus surgery and anatomy. It is considered that there are significant differences of the sinuses between individuals, but even for the same individual, between the right and left sides. The development process of the sinuses can be affected by genetic factors, environmental conditions and past infections ${ }^{1}$. There are multiple studies and publications related to anatomical variations of the sinuses, and their development, but less about dimensions and volumes. Once endoscopic sinus surgery has evolved and is nowadays the gold standard surgical treatment for most types of sinus pathology, the need for detailed studies has increased. It is considered crucial for the sinus surgeon to have an extensive knowledge on the region, especially prior to performing procedures ${ }^{1-5}$. Sinus volumes are not commonly measured in practice since it is not always possible, and, if possible, it could involve too much ef- fort $^{1,6}$. Due to this fact, we found little data on sinus volumes, especially for the ethmoid sinus. In this paper, we present data from our own volumetric study regarding the ethmoid lateral mass, as well as the volumes of the anterior and posterior ethmoids.

\section{MATERIAL AND METHODS}

For this study, we used a number of 150 native computed-tomography (CT) examinations (300 lateral ethmoid masses). These were randomly chosen from our database. We did not take the sex and the age of the individual as a variable in the study. We excluded from the study patients that underwent prior surgery of the paranasal sinuses, patients with potential destructive pathology and computed-tomography studies without proper resolution.

For each CT we measured individually the volumes of the two ethmoid lateral masses, and after that the volumes of the anterior and posterior ethmoid groups. 
The limit between the anterior and posterior ethmoid groups was considered to be the basal lamella of the middle turbinate. We also considered as part of the lateral masses of the ethmoid the different extramural cells (Haller's cells, Onodi cells, etc).

The measurements were performed using the dedicated OsiriX software and its volumetric function. This is based on the Cavalieri principle, which automatically generates and calculates a volume, using seriated measurements of the area in different planes of section. The volume was calculated in $\mathrm{cm}^{3}$ and a $3 \mathrm{D}$ diagram of the volume was generated (Figure 1). After all the measurements were performed, the data were analyzed and we noted the minimal and the maximal values, we calculated the average value for each volume, and the standard deviation (SD). The values were then compared between the anterior and posterior groups, and between the right and left ethmoid lateral masses. The "t-test" was used to check if the differences between data were statistically accurate.

\section{RESULTS}

For each ethmoid lateral mass we calculated, on each side, the volume of the whole lateral mass, and of the anterior and posterior ethmoid groups.

For the left ethmoid, we calculated an average volume of $8.39 \mathrm{~cm}^{3}$ with a $1.99 \mathrm{SD}$. The minimal volume of the left ethmoid lateral mass was $4.47 \mathrm{~cm}^{3}$ and the maximal was $12.46 \mathrm{~cm}^{3}$ (Table 1). For the anterior left ethmoid, the average volume was $4.92 \mathrm{~cm}^{3}$ with a $2.11 \mathrm{SD}$. The minimal volume was of $0.26 \mathrm{~cm}^{3}$ and a maximal of $10.38 \mathrm{~cm}^{3}$.

For the posterior left ethmoid, we found an average volume of $3.47 \mathrm{~cm}^{3}$ with a $0.90 \mathrm{SD}$, a minimal and a maximal value of $1.88 \mathrm{~cm}^{3}$ and $5.45 \mathrm{~cm}^{3}$ respectively.

For the right ethmoid, we found that the average value of the volume was $7.34 \mathrm{~cm}^{3}$ with a $1.51 \mathrm{SD}$. The minimal and maximal volumes were $4.55 \mathrm{~cm}^{3}$ and $10.60 \mathrm{~cm}^{3}$ respectively (Table 2). The anterior right ethmoid had an average volume of $4.33 \mathrm{~cm}^{3}$ with a $1.63 \mathrm{SD}$. The minimal and maximal volumes were $0.62 \mathrm{~cm}^{3}$ and $7.79 \mathrm{~cm}^{3}$

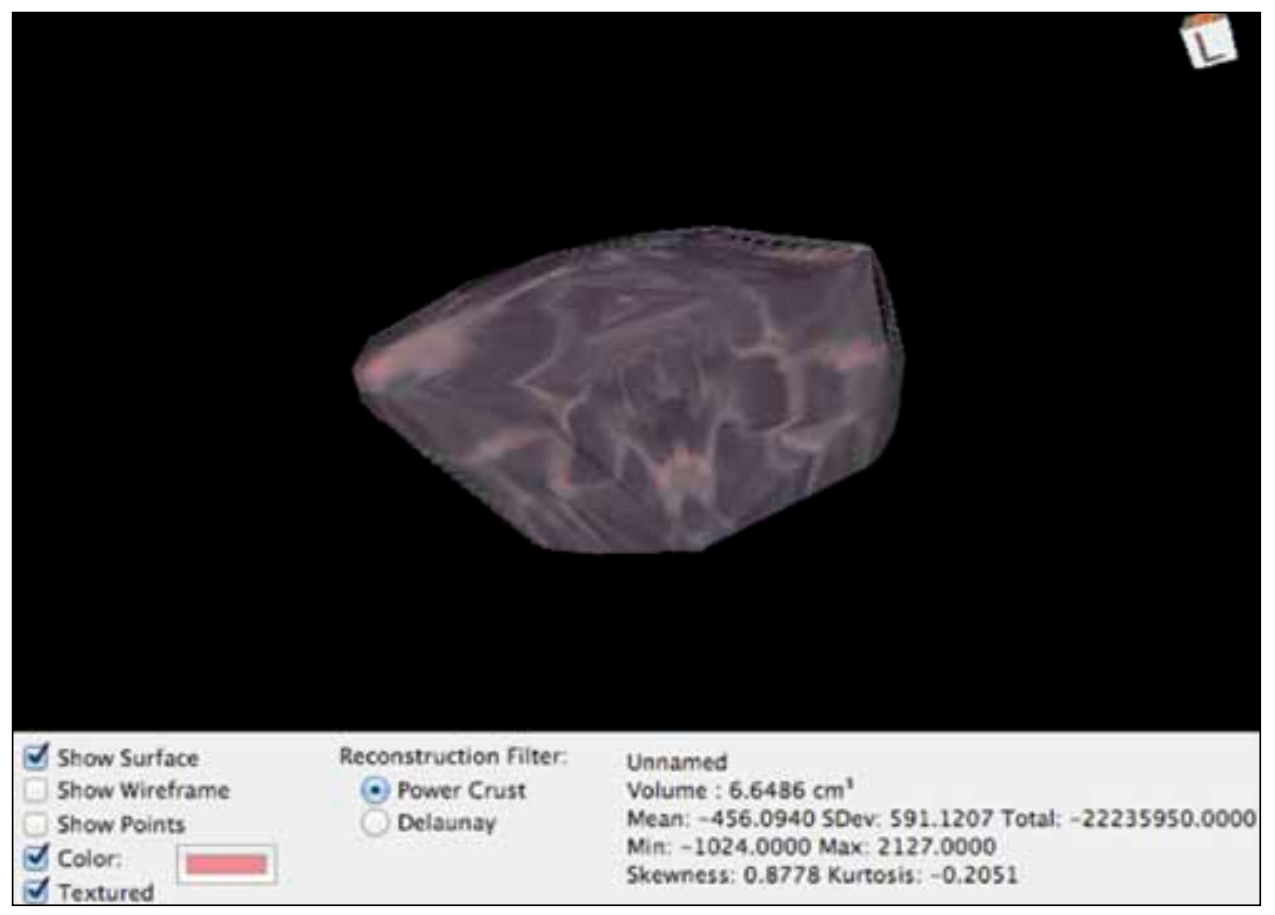

Figure $13 \mathrm{D}$ diagram reconstruction of a right ethmoid lateral mass.

Table 1

Volumetric data of the left ethmoid lateral mass

\begin{tabular}{cccc}
\hline Data & Left ethmoid lateral mass & Left anterior ethmoid & Left posterior ethmoid \\
\hline Average value $(\mathrm{cm} 3)$ & 8.39 & 4.92 & 3.47 \\
\hline Minimal value $(\mathrm{cm} 3)$ & 4.47 & 0.26 & 1.88 \\
\hline Maximal value $(\mathrm{cm} 3)$ & 12.46 & 10.38 & 5.45 \\
\hline Standard deviation & 1.99 & 2.11 & 0.90 \\
\hline
\end{tabular}


Table 2

Volumetric data of the right ethmoid lateral mass

\begin{tabular}{cccc}
\hline Data & Right ethmoid lateral mass & Right anterior ethmoid & Right posterior ethmoid \\
\hline Average value $(\mathrm{cm} 3)$ & 7.34 & 4.33 & 3.01 \\
\hline Minimal value $(\mathrm{cm} 3)$ & 4.55 & 0.62 & 1.66 \\
\hline Maximal value $(\mathrm{cm} 3)$ & 10.60 & 7.79 & 4.36 \\
\hline Standard deviation & 1.51 & 1.63 & 0.75 \\
\hline
\end{tabular}

respectively. The posterior right ethmoid had an average volume of $3.01 \mathrm{~cm}^{3}$ with a $0.75 \mathrm{SD}$, a minimal value of $1.66 \mathrm{~cm}^{3}$ and a $4.36 \mathrm{~cm}^{3}$ maximal volume.

Analyzing the data we found that for both right and left lateral masses of the ethmoid, there was a significant difference between the volumes of the anterior and posterior ethmoid groups in relation to the volume of the whole lateral mass.

The average volume of the left ethmoid was 8.39 $\mathrm{cm}^{3}$, while the average volumes of the left anterior and posterior ethmoid groups were $4.92 \mathrm{~cm}^{3}$ and $3.47 \mathrm{~cm}^{3}$. Observing these, we calculated that the average volume of the anterior group represents $58.46 \%$ of the whole volume, while the posterior group is $41.35 \%$ of this volume. The "t-test" showed the data were statistically significant $(\mathrm{t}=8.989, \mathrm{p}<0.05)$.

The same percentages were calculated for the right ethmoid. In this case, the average volume of the lateral mass was $7.34 \mathrm{~cm}^{3}$, while average volumes of the anterior and posterior groups were $4.33 \mathrm{~cm}^{3}$ and $3.01 \mathrm{~cm}^{3}$. Our calculations showed that the volume of the anterior ethmoid group represents $58.99 \%$, and the one of the posterior ethmoid group is $41.008 \%$. The data were also statistically significant $(\mathrm{t}=8.989, \mathrm{p}<0.05)$.

After calculating the volumes of each ethmoid group, we observed that there is no statistically significant difference between the volumes of the right and left ethmoid lateral masses $(\mathrm{t}=5.118, \mathrm{p}>0.05)$.

The same was observed for the anterior groups $(\mathrm{t}$ $=2.694, \mathrm{p}>0.05)$ and posterior groups $(\mathrm{t}=4.76, \mathrm{p}>0.05)$.

\section{DISCUSSIONS}

The roles and importance of paranasal sinuses is not yet clear, being still in a state of hypothesis. Most of the features postulated as functions of the sinuses, such as warming and dampening the air during inspiration, or acting as resonance cavities during speech, are still to be proven ${ }^{1}$. Even so, the clinical importance of the sinuses is clear, since they are often sites for chronic infection, such as chronic sinusitis.

The ethmoid sinus is probably the most interesting, controversial and important of the paranasal sinuses.
From an embryologic point of view, the ethmoid is different from all other paranasal sinuses. It originates from the cartilaginous nasal capsule (paleosinus), while the other paranasal sinuses will develop as extensions from the capsule (extracapsular migration) into the surrounding bones?

In anatomical terms, the ethmoid is also different from other paranasal sinuses, due to the fact that its cells are separated by very thin bony lamellae, while the walls of other sinuses are more rigid and robust. Due to this important characteristic of ethmoid cells, it is said that one cell may outgrow the ones next to it and force them in another direction, or limit their expansion $^{7,8}$. Different pneumatization and expansion types of the ethmoid make it a challenge to establish a certain pattern in its anatomy.

Knowledge about the normal and average size and volume of paranasal sinuses could serve as an indication and point of reference for abnormal anatomy. Therefore, the idea of the study was to offer an estimate to the average sizes of the ethmoid lateral mass, serving as a guideline for surgeons and anatomists.

Volumetric studies of paranasal sinuses were performed and can be found in literature, using several methods and imaging. Some of these studies used measurements of distances between sinus walls, and calculated the volume of the sinuses by trying to resemble the shape of the sinus to a geometrical shape ${ }^{9}$. Other more recent papers have published reports using the Cavalieri principle on imaging studies. This formula uses two-dimensional images from imaging studies in order to get a three-dimensional volume ${ }^{10,11}$. Aside from the techniques using imaging series, other studies have been made using cadavers or dry skulls ${ }^{12,13}$. We consider that there is not a perfect method of measuring volumes of the paranasal sinuses, since these are irregular in shape and imaging studies are not always as accurate as they should be.

In our study, we used a feature of OsiriX, which generated a 3D diagram and calculated the volume after we selected the area of the ethmoid on each transverse section from the imaging study. We did the same thing for both right and left ethmoid cells, and also for each one of them we measured the anterior and posterior groups. 
In order to separate anterior ethmoid cells from the posterior ones, we used the basal lamella of the middle turbinate as a landmark. This was proved to be difficult in some cases when some cells of the posterior ethmoid bent the basal lamella and were situated superior to the anterior ones. In these cases, we found rather irregular shaped ethmoid groups. The method is also not perfect since the accuracy of the $3 \mathrm{D}$ reconstruction is dependent on the thickness of imaging slices.

In our study, we did not compare the volumes of ethmoid sinuses between male and female groups, considering that there would not be a significant difference between the two. Some studies reported a difference in paranasal sinus volumes between males and female, sinuses being larger in males. ${ }^{1,13,14}$. Other papers showed no statistically significant differences between male and female paranasal sinuses ${ }^{15-16}$.

Regarding the volume of the ethmoid sinus, we found few studies that measured this parameter, probably due to its important variability and irregular shape. The average volume of the ethmoid sinus would vary between $5.5+/-1.5 \mathrm{~cm}^{3}$ in females and $6.3+/-1.6 \mathrm{~cm}^{3}$ in males according to M. Emirzeoglu et al. ${ }^{1}$, and $5.08+/-2.83 \mathrm{~cm}^{3}$ on the right side and $4.77+/-$ $2.04 \mathrm{~cm}^{3}$ on the left side according to Amusa et al. ${ }^{17}$.

Our results showed an average volume of the ethmoid sinus of $8.39+/-1.99 \mathrm{~cm}^{3}$ on the left side and $7.34+/-1.51 \mathrm{~cm}^{3}$ on the right side. The small difference between sides was not statistically significant $(\mathrm{t}=5.118, \mathrm{p}>0.05)$.

In our study, we also measured the volumes of the anterior and posterior ethmoid groups and after that we calculated the percentage of each group from the total volume of the ethmoid. We found that with statistical significance we can state that the anterior ethmoid occupies $58-59 \%$ of the whole ethmoid lateral mass, while the posterior ethmoid group occupies the rest of $41-42 \%$. We did not find any other papers that calculated the volumes of anterior and posterior ethmoid groups. This could be important information for sinus surgeons when planning their technique.

\section{CONCLUSIONS}

Knowledge of paranasal sinus anatomy has become crucial with the evolution of surgical techniques and increased practice. The anatomy of the ethmoid sinus is, and probably will always be, challenging, since it is highly variable and dependent on many other structures. The innovation of this paper was the volumetric measurement of anterior and posterior ethmoid cells as well as the entire volume of the ethmoid. The calculations we made concluded that the ethmoid lateral mass is divided into $58-59 \%$ for the anterior ethmoid and $41-42 \%$ for the posterior ethmoid.
Conflict of interest: The authors have no conflict of interest.

Contribution of authors: All authors have equally contributed to this work.

\section{REFERENCES}

1. Emirzeoglu M., Sahin B., Bilgic S., Celebi M., Uzun A. - Volumetric evaluation of paranasal sinuses in normal subjects using computer tomography images: a stereological study. Auris Nasus Larynx, 2007;34(2):191195. Epub 2006 Nov 3.

2. Stammberger H.R., Kennedy D.W.; Anatomic Terminology Group. Paranasal sinuses: anatomic terminology and nomenclature. Ann Otol Rhinol Laryngol Suppl., 19985;167:7-16.

3. Zinreich S.J., Mattox D.E., Kennedy D.W., Chisholm H.L., Diffley D.M., Rosenbaum A.E. - Concha bullosa: CT evaluation. J Comput Assist Tomogr., 1988;12(5):778-784.

4. Unlu H.H., Akyar S., Caylan R., Nalca Y. - Concha bullosa. J Otolaryngol., 1994;23:23-27.

5. Gunkel A.R., Freysinger W., Thumfart W.F. - 3D anatomo-radiological basis of endoscopic surgery of the paranasal sinuses. Surg Radiol Anat., 1997;19(1):7-10.

6. Ariji Y., Ariji E., Yoshiura K., Kanda S. - Computed tomographic indices for maxillary sinus size in comparison with the sinus volume. Dentomaxilofac Radiol., 1996;25(1):19-24.

7. Marquez S., Tessema B., Clement P.A., Schaefer S.D. - Development of the ethmoid sinus and extramural migration: the anatomical basis of this paranasal sinus. Anat Rec (Hoboken), 2008;291(11):1535-1553. doi: 10.1002/ar.20775.

8. Anon J.B., Zinreich S.J., Rontal M. - Anatomy of the paranasal sinuses. Thieme Medical Publiaher, New York, 1996.

9. Bargbouth G., Prior J.O., Lepori D., Duvoisin B., Schnyder P., Gudinchet F. - Paranasal sinuses in children: size evaluation of maxillary, sphenoid, and frontal sinuses using magnetic resonance imaging and proposal of volume index percentile curves. Eur Radiol., 2002;12(6):1451-1458. Epub 2002 Feb 2.

10. Emirzeoglu M., Sahin B., Selcuk M.B., Kaplan S. - The effects of section thickness on the estimation of live volume by the Cavalieri principle using computed-tomography images. Eur J Radiol., 2005;56(3):391-397.

11. Sahin B., Emirzeoglu M., Uzun A., Incesu L., Bek Y., Bilgic S., Kaplan S. - Unbiased estimation of the liver volume by the Cavalieri principle using magnetic resonance images. Eur J Radiol., 2003;47(2):164-170.

12. Anagnostopoulou S., Venieratos D., Spyropoulos N. - Classification of human maxillary sinuses according to their geometric features. Anat Anz., 1991;173(3):121-130.

13. Uchida Y., Goto M., Katsuki T., Akiyoshi T. - A cadaveric study of maxillary sinus sizes aid in bone grafting of the maxillary sinus floor. J Oral Maxilofac Surg., 1998;56(10):1158-1163.

14. Karakas S., Kavakli A. - Morphometric examination of paranasal sinuses and mastoid air cells using computed-tomography. Ann Saudi Med., 2005;25(1):41-45.

15. Kawarai Y., Fukushima K., Ogawa T., Nishizaki K., Gunduz M., Fujimoto M., Masuda Y. - Volume quantification of healthy paranasal cavity by three-dimensional CT imaging. Acta Otolaryngol Suppl., 1999;540:45-49.

16. Sanchez Fernandez J.M., Anta Escuredo J.A., Sanchez Del Rey A., Santaolalla Montoya F. - Morphometric study of paranasal sinuses in normal and pathological conditions. Acta Otolaryngol., 2000;120(2):273-278.

17. Amusa Y.B., Eziyi J.A.E., Akinlade O., Famurewa O.C., Adewole S.A., Nwoha P.U., Ameye S.A. - Volumetric measurements and anatomical variants of paranasal sinuses of Africans (Nigerians) using dry crania. International Journal of Medicine and Medical Sciences, 2011;3(10):299-303. 